\title{
Capacidade funcional e sua mensuração em idosos: uma revisão integrativa
}

\author{
Functional capacity and its measurement in the elderly: an integrative review \\ Capacidad funcional y su medición en el anciano: una revisión integradora
}

Recebido: 01/08/2013

Aprovado: 21/03/2014
Gerson de Souza Santos ${ }^{1}$

Isabel Cristina Kowal Olm Cunha2

O objetivo deste estudo foi buscar evidências científicas que abordem a capacidade funcional em idosos, bem como instrumentos utilizados para mensurá-la. Trata-se de uma revisão integrativa da literatura, na qual se incluíram artigos indexados nas bases de dados LILACS e SCIELO publicados em língua portuguesa no Brasil de 2008 a 2012. 0 ano de publicação dos artigos variou entre 2010 e 2012, havendo predominância do estudo do tipo transversal. Os profissionais enfermeiros foram os principais responsáveis pelas publicações. Questionários e escalas foram utilizados para avaliar a capacidade funcional dos idosos, com predominância do Índice de Katz, sendo a grande maioria dos artigos provenientes de grupos de pesquisa de universidades públicas. As publicações demonstraram que, por meio da utilização de escalas e questionários, é possível identificar os fatores que limitam a capacidade funcional dos idosos.

Descritores: Enfermagem; Idoso; Saúde coletiva.

The aim of this study was to seek scientific evidence that address the functional capacity in the elderly as well as instruments used to measure it. It is an integrative review of the literature, which included articles indexed in the LILACS and SCIELO databases, published in Portuguese in Brazil from 2008 to 2012. The year of publication of the studies ranged between 2010 and 2012, with predominance of the cross-sectional study. The nurses were primarily responsible for publications. Questionnaires and scales were used to assess the functional capacity of the elderly, with predominance of Katz Index. Most of the articles are from research groups of public universities. Publications have demonstrated that through the use of scales and questionnaires, it is possible to identify the factors that limit the functional capacity of the elderly.

Descriptors: Nursing; Aged; Public health.

El objetivo de este estudio fue buscar evidencia científica acerca de la capacidad funcional de ancianos, así como los instrumentos utilizados para medirla. Se trata de una revisión integradora de la literatura, que incluyeron artículos indizados en las bases de datos LILACS y SCIELO publicado en portugués en Brasil de 2008 a 2012. El año de la publicación de los estudios varió entre 2010 y 2012 con predominio de estudios transversales. Las enfermeras eran las principales responsables de las publicaciones. Cuestionarios y escalas se utilizaron para evaluar la capacidad funcional de los ancianos, sobre todo Katz Index. La mayor parte de los artículos son de grupos de investigación de universidades públicas. Las publicaciones demostraron que a través de la utilización de escalas y cuestionarios, es posible identificar los factores que limitan la capacidad funcional de los ancianos.

Descriptores: Enfermería; Anciano; Salud colectiva.

\footnotetext{
${ }^{1}$ Enfermeiro. Doutorando Escola Paulista de Enfermagem pela Universidade Federal de São Paulo - UNIFESP. Grupo de Estudo em Pesquisa e Pesquisa em Administração de Serviços de Saúde e Gerenciamento de Enfermagem - GEPAG. gersonenf@hotmail.com

2 Enfermeira. Professora Livre Docente na UNIFESP. Líder do GEPAG.
} 


\section{INTRODUÇÃO}

População mundial está

A envelhecendo. Estudos mostram que o número de pessoas idosas cresce em ritmo maior do que o de pessoas que nascem, acarretando um conjunto de situações que modificam a estrutura de gastos dos países em diferentes áreas. No Brasil, o ritmo de crescimento da população idosa tem sido sistemático e consistente. No período de 1999 a 2009, o peso relativo dos idosos (60 anos ou mais de idade) no conjunto da população passou de 9,1\% para $11,3 \%$. Combinada ainda com outros fatores, tais como os avanços da tecnologia, especialmente na área da saúde, atualmente o grupo de idosos ocupa um espaço significativo na sociedade brasileira ${ }^{1}$.

Nesse sentido, o Instituto Brasileiro de Geografia e Estatística (IBGE) vêm alertando, por meio dos indicadores sociais e demográficos divulgados anualmente, que a estrutura etária do país está mudando e que o grupo de idosos é, hoje, um contingente populacional expressivo em termos absolutos e de crescente importância relativa no conjunto da sociedade brasileira, decorrendo daí uma série de novas exigências e demandas em termos de políticas públicas de saúde e inserção ativa dos idosos na vida social, bem como da avaliação de sua capacidade funcional ${ }^{1}$.

À medida que um maior número de pessoas atinge idades mais avançadas, há uma tendência de alteração no padrão de morbidade e de causas de morte da população. Assim, ao invés das doenças infectocontagiosas as doenças crônicodegenerativas e suas complicações tornaram-se predominantes. A tendência atual é ter um número crescente de indivíduos idosos que, apesar de viverem mais, experimentam maior número de condições crônicas ${ }^{2}$.

A curto e longo prazos, o aumento no número de doenças crônicas leva a uma maior prevalência de incapacidade funcional. Ademais, com o processo fisiológico do envelhecimento, a capacidade funcional de cada sistema do organismo humano diminui.
Trata-se de um processo lento e imperceptível, mas inexorável e universal2 ${ }^{2}$.

A avaliação da capacidade funcional torna-se essencial para o estabelecimento de um diagnóstico, um prognóstico e um julgamento clínico adequados, que servirão de base para as decisões sobre os cuidados necessários às pessoas idosas. É um parâmetro que, associado a outros indicadores de saúde, pode ser utilizado para determinar a efetividade e a eficiência das intervenções propostas. A avaliação funcional busca verificar, de forma sistematizada, em que nível as doenças ou agravos impedem o desempenho, de forma autônoma e independente, das atividades cotidianas ou atividades de vida diária (AVD) das pessoas idosas, permitindo o desenvolvimento de um planejamento assistencial mais adequado ${ }^{2,3}$.

Muitos modelos de avaliação funcional foram desenvolvidos e aplicados nas últimas décadas em várias categorias populacionais. Os modelos são criados e selecionados para categorias que apresentam a mesma especificidade: indivíduos mais ou menos dependentes, institucionalizados, hospitalizados ou da comunidade, portadores de patologias específicas (tais como artrite, osteoartrite, doença de Parkinson, acidente vascular cerebral e outras). A maioria desses testes é pouco sensível às pequenas perdas funcionais e não é projetada para indivíduos idosos hígidos que apresentam pequenos déficits do declínio fisiológico ${ }^{3,4}$.

0 presente artigo teve por objetivo buscar evidências científicas que abordem a capacidade funcional em idosos, bem como instrumentos utilizados para mensurá-la, através de artigos científicos.

\section{MÉTODO}

Esta é uma revisão integrativa, que inclui a análise de pesquisas relevantes que dão suporte para a tomada de decisão, permitindo a incorporação desses achados na prática clínica. Esse tipo de estudo é uma estratégia para a identificação e análise das evidências existentes de práticas de saúde, 
quando a produção de conhecimento científico não está suficientemente fundamentada. Para a elaboração de uma revisão integrativa, faz-se necessária a adoção de fases que apresentem um rigor metodológico em busca de evidências sobre determinado assunto. Para a operacionalização dessa revisão integrativa, utilizou-se os seguintes passos metodológicos: definição dos critérios de inclusão e exclusão; definição das informações a serem extraídas dos estudos selecionados; categorização dos estudos; análise e interpretação dos dados; avaliação dos resultados incluídos na revisão integrativa e apresentação da revisão/síntese do conhecimento ${ }^{5}$.

A busca bibliográfica foi realizada nas bases de dados de Literatura LatinoAmericana e do Caribe em Ciências da Saúde (LILACS) e Scientific Electronic Library Online (SCIELO). Foram utilizados os Descritores em Ciências da Saúde (DeCS): "capacidade funcional", "idosos", "funcionalidade". Os artigos foram selecionados de acordo com os seguintes critérios de inclusão: presença dos descritores escolhidos no título do trabalho ou inseridos no resumo, artigos na íntegra, produções com idioma em português, originárias no Brasil e publicadas entre janeiro de 2008 e dezembro de 2012. A busca foi realizada no mês de janeiro de 2013. Foram encontrados 225 artigos indexados na base de dados LILACS e 373 artigos na base de dados SCIELO. Destes, apenas 20 atenderam aos critérios de inclusão para responder ao objetivo deste estudo.

Foram excluídos desta pesquisa os artigos em forma de apostilas, cartas e editoriais, pois não contemplavam os critérios necessários para uma pesquisa científica, visto que o foco deste estudo era buscar evidências científicas sobre 0 assunto.

Para catalogar os artigos e realizar uma posterior avaliação, foi elaborado um instrumento de coleta de dados. 0 instrumento foi composto por: nome do periódico, ano de publicação, área de conhecimento, vínculo institucional do autor, 270 origem do artigo, título do artigo, objetivos, tipo de estudo, características dos idosos, instrumentos e variáveis estudadas.

As análises foram realizadas por meio de leitura e agrupamento dos $\operatorname{artigos}^{5}$ e alicerçadas no instrumento elaborado e na seleção por meio dos critérios de inclusão e exclusão. Os resultados foram apresentados na forma de quadros e na linguagem descritiva. Para melhor visualização, optouse por separar duas áreas de discussão, descritas a seguir: capacidade funcional e envelhecimento e instrumentos utilizados para mensuração da capacidade funcional em idosos.

\section{RESULTADOS}

Dos 20 artigos publicados sobre o tema em questão, percebeu-se nesta revisão integrativa que estes foram publicados nos seguintes periódicos: três (15\%) na Revista Acta Paulista de Enfermagem; dois (10\%) na Revista Latino-Americana de Enfermagem; dois (10\%) na Revista Brasileira de Ciências da Saúde; dois (10\%) na Revista Ciência e Saúde Coletiva; dois (10\%) na Revista Baiana de Saúde Pública; um (5\%) na Revista Cogitare Enfermagem; um (5\%) na Revista Brasileira de Enfermagem; um (5\%) na Revista Gaúcha de Enfermagem; um (5\%) na Revista de Rede de Enfermagem do Nordeste; e um (5\%) na Revista Texto e Contexto Enfermagem; um (5\%) nos Cadernos de Saúde Pública; um (5\%) no Caderno de Terapia Ocupacional; um (5\%) na Revista Brasileira de Fisioterapia; um (5\%) na Revista de Saúde Pública; apresentados no Quadro 1.

Em relação ao ano de publicação dos artigos, constatou-se que sete (35\%) são do ano 2010; cinco (25\%) do ano 2011; quatro (20\%) de 2012; dois (10\%) do ano 2009 e dois (10\%) do ano 2008.

Concernente ao vínculo do autor responsável, destaca-se que todos pertencem a instituições públicas de ensino, sendo nove (45\%) da Região Sudeste; cinco (25\%) da Região Sul; cinco (25\%) da Região Nordeste e um (5\%) da Região Centro-Oeste. Dos artigos encontrados 15 (83\%) são de grupos de pesquisas e cinco (17\%) REFACS(online)2014;2(3):269-278 
produzidos com base em dissertação de mestrado. Todos os estudos foram pesquisas quantitativas com abordagens variadas, como: 15 (75\%) do tipo transversal; dois (10\%) do tipo seccional; um (5\%) do tipo Quadro 1 - Artigos segundo periódico, ano de publicação, área do conhecimento, vínculo institucional e origem do artigo. São Paulo, SP, 2013.

\begin{tabular}{|c|c|c|c|c|}
\hline $\begin{array}{c}\text { Nome do } \\
\text { periódico }\end{array}$ & Ano & $\begin{array}{c}\text { Área de } \\
\text { conhecimento }\end{array}$ & Vínculo institucional & Origem do artigo \\
\hline $\begin{array}{lll}\text { Ciência } \\
\text { Coletiva }\end{array}$ & 2008 & Enfermagem & $\begin{array}{l}\text { Universidade Federal de } \\
\text { Goiás }\end{array}$ & Grupo de Pesquisa \\
\hline $\begin{array}{l}\text { Acta Paulista de } \\
\text { Enfermagem }\end{array}$ & 2008 & Enfermagem & Universidade de São Paulo & Grupo de Pesquisa \\
\hline $\begin{array}{lll}\text { Ciência } & \text { e } & \text { Saúde } \\
\text { Coletiva } & & \\
\end{array}$ & 2009 & Fisioterapia & $\begin{array}{ll}\text { Universidade Federal do } \\
\text { Triângulo Mineiro }\end{array}$ & Grupo de Pesquisa \\
\hline Rev. Bras Fisioter. & 2009 & Fisioterapia & $\begin{array}{l}\text { Universidade Federal de } \\
\text { Viçosa-MG }\end{array}$ & Grupo de Pesquisa \\
\hline $\begin{array}{l}\text { Rev. Gaúcha de } \\
\text { Enferm. }\end{array}$ & 2010 & Enfermagem & $\begin{array}{l}\text { Universidade Federal do Rio } \\
\text { Grande do Sul }\end{array}$ & $\begin{array}{l}\text { Trabalho de Conclusão de } \\
\text { Curso }\end{array}$ \\
\hline $\begin{array}{l}\text { Rev. Latino-Am. de } \\
\text { Enfermagem }\end{array}$ & 2010 & Enfermagem & $\begin{array}{l}\text { Universidade Federal do Rio } \\
\text { Grande do Sul }\end{array}$ & Grupo de pesquisa \\
\hline $\begin{array}{l}\text { Cogitare } \\
\text { Enfermagem }\end{array}$ & 2010 & Enfermagem & Universidade de São Paulo & Grupo de Pesquisa \\
\hline $\begin{array}{l}\text { Revista Baiana de } \\
\text { Saúde Pública }\end{array}$ & 2010 & Enfermagem & Universidade de São Paulo & Grupo de Pesquisa \\
\hline $\begin{array}{l}\text { Acta Paulista de } \\
\text { Enfermagem }\end{array}$ & 2010 & Enfermagem & Universidade de São Paulo & Grupo de Pesquisa \\
\hline $\begin{array}{lll}\text { Ciência } & \text { e } & \text { Saúde } \\
\text { Coletiva } & & \\
\end{array}$ & 2010 & Enfermagem & $\begin{array}{ll}\text { Universidade } & \text { Estadual de } \\
\text { Santa Catarina } & \end{array}$ & Grupo de Pesquisa \\
\hline $\begin{array}{l}\text { Revista Baiana de } \\
\text { Saúde Pública }\end{array}$ & 2010 & Fisioterapia & $\begin{array}{l}\text { Universidade Estadual do } \\
\text { Sudoeste da Bahia }\end{array}$ & Grupo de Pesquisa \\
\hline $\begin{array}{l}\text { Acta Paulista de } \\
\text { Enfermagem }\end{array}$ & 2011 & Enfermagem & $\begin{array}{l}\text { Universidade Federal de São } \\
\text { Carlos }\end{array}$ & Dissertação de Mestrado \\
\hline Rev. Rene & 2011 & Enfermagem & $\begin{array}{l}\text { Universidade } \text { Estadual de } \\
\text { Londrina }\end{array}$ & PIBIC \\
\hline $\begin{array}{l}\text { Rev. Brasileira de } \\
\text { Ciências da Saúde }\end{array}$ & 2011 & Enfermagem & $\begin{array}{lll}\text { Universidade } & \text { Federal da } \\
\text { Paraíba } & & \\
\end{array}$ & Grupo de Pesquisa \\
\hline $\begin{array}{l}\text { Rev. Brasileira de } \\
\text { Ciências da Saúde }\end{array}$ & 2011 & Fisioterapia & $\begin{array}{lll}\text { Universidade } & \text { Federal da } \\
\text { Paraíba } & & \\
\end{array}$ & Grupo de Pesquisa \\
\hline $\begin{array}{l}\text { Revista de Saúde } \\
\text { Pública }\end{array}$ & 2011 & $\begin{array}{l}\text { Educação } \\
\text { Física }\end{array}$ & $\begin{array}{lll}\text { Universidade } & \text { Federal de } \\
\text { Santa Catarina } & & \\
\end{array}$ & Grupo de Pesquisa \\
\hline $\begin{array}{l}\text { Rev. Bras. de } \\
\text { Enferm. }\end{array}$ & 2012 & Enfermagem & $\begin{array}{lll}\text { Universidade } & \text { Federal de } \\
\text { Minas Gerais } & & \\
\end{array}$ & Grupo de Pesquisa \\
\hline $\begin{array}{l}\text { Rev. Latino-Am. de } \\
\text { Enfermagem }\end{array}$ & 2012 & Enfermagem & $\begin{array}{l}\text { Universidade Federal de São } \\
\text { Carlos }\end{array}$ & Dissertação de Mestrado \\
\hline $\begin{array}{l}\text { Texto Contexto } \\
\text { Enfermagem }\end{array}$ & 2012 & Enfermagem & $\begin{array}{lll}\text { Universidade } & \text { Federal da } \\
\text { Paraíba } & & \\
\end{array}$ & Dissertação de Mestrado \\
\hline $\begin{array}{l}\text { Cad. Ter. Ocup. } \\
\text { UFSCar }\end{array}$ & 2012 & Fisioterapia & $\begin{array}{l}\text { Universidade Estadual de } \\
\text { Ciências de Alagoas }\end{array}$ & Grupo de Pesquisa \\
\hline
\end{tabular}

prospectivo; um (5\%) do tipo multicêntrico; e um (5\%) do tipo exploratório, sendo que os profissionais enfermeiros foram responsáveis por $70 \%$ das publicações da temática em questão. 
pesquisadores de conhecer o idoso na sua totalidade e relacionarem como as características socioeconômicas e demográficas influenciariam na capacidade funcional e nas condições de saúde dos idosos. Quanto aos instrumentos utilizados para a coleta de dados, o questionário foi o mais utilizado para dados referentes às características socioeconômicas e demográficas.
Em relação aos instrumentos utilizados para mensuração da capacidade funcional, 10 (50\%) utilizaram o Índice de Katz; quatro (20\%) o Índice de Barthel; três (15\%) o instrumento Older Americans Resourses and Services (OARS); dois (10\%) a Medida de Independência Funcional (MIF); e um (5\%) a Escala de Lawton, conforme o Quadro 2.

Quadro 02 - Artigos segundo título, tipo de estudo, objetivo e instrumento utilizado para a avaliação da capacidade funcional. São Paulo, SP, 2013.

\begin{tabular}{|c|c|c|c|}
\hline Título & $\begin{array}{l}\text { Tipo de } \\
\text { estudo }\end{array}$ & Objetivo & $\begin{array}{l}\text { Instrumento de } \\
\text { mensuração }\end{array}$ \\
\hline $\begin{array}{l}\text { Prevalência e fatores associados ao } \\
\text { cuidado domiciliar a idosos. }\end{array}$ & Transversal & $\begin{array}{l}\text { Estimar a prevalência do cuidado } \\
\text { domiciliar prestado aos idosos e identificar } \\
\text { fatores associados. }\end{array}$ & Índice de Katz \\
\hline $\begin{array}{l}\text { Índice de Katz aplicado a idosos } \\
\text { institucionalizados. }\end{array}$ & Transversal & $\begin{array}{l}\text { Avaliar o perfil e o grau de dependência de } \\
\text { idosos institucionalizados residentes em } \\
\text { Instituições Filantrópicas de longa } \\
\text { permanência. }\end{array}$ & Índice de Katz \\
\hline $\begin{array}{l}\text { Capacidade } \\
\text { Funcional de idosos } \\
\text { adscritos à Estratégia Saúde da } \\
\text { Família no município de João } \\
\text { Pessoa-PB. }\end{array}$ & Transversal & $\begin{array}{l}\text { Traçar o perfil funcional e identificar quais } \\
\text { fatores estão associados à capacidade para } \\
\text { a realização das ABVD de uma amostra } \\
\text { representativa de idosos adscritos à } \\
\text { Estratégia "Saúde da Família" (ESF) do } \\
\text { município de João Pessoa-PB. }\end{array}$ & Índice de Katz \\
\hline $\begin{array}{l}\text { Capacidade funcional de idosos mais } \\
\text { velhos: um estudo comparativo em } \\
\text { três regiões do Rio Grande do Sul. } \\
\end{array}$ & Transversal & $\begin{array}{l}\text { Comparar o grau de dependência para as } \\
\text { AVDs em idosos com } 80 \text { anos ou mais em } \\
\text { três regiões do Rio Grande do Sul. }\end{array}$ & Índice de Katz \\
\hline $\begin{array}{l}\text { Capacidade funcional, condições } \\
\text { socioeconômicas e de saúde de } \\
\text { idosos atendidos por equipes de } \\
\text { Saúde da Família de Goiânia-GO. }\end{array}$ & Transversal & $\begin{array}{l}\text { Avaliar a capacidade funcional, identificar } \\
\text { os fatores associados à dependência e } \\
\text { descrever os perfis socioeconômico, } \\
\text { demográfico e de saúde de idosos. }\end{array}$ & Índice de Katz \\
\hline $\begin{array}{l}\text { Funcionalidade de idosos com } \\
\text { alterações cognitivas em diferentes } \\
\text { contextos de vulnerabilidade social. }\end{array}$ & Transversal & 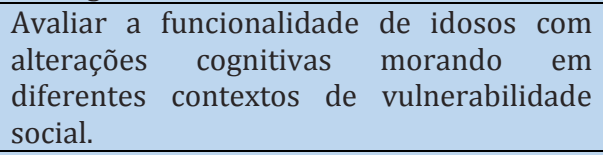 & Índice de Katz \\
\hline $\begin{array}{l}\text { Relação entre apoio social e } \\
\text { capacidade funcional de idosos com } \\
\text { alterações cognitivas. }\end{array}$ & Transversal & $\begin{array}{l}\text { Identificar a relação entre apoio social e } \\
\text { capacidade funcional de idosos com } \\
\text { alterações cognitivas cadastrados em } \\
\text { diferentes Unidades de Saúde da Família. }\end{array}$ & Índice de Katz \\
\hline $\begin{array}{l}\text { Perfil epidemiológico, clínico e de } \\
\text { independência funcional de uma } \\
\text { população idosa institucionalizada. }\end{array}$ & $\begin{array}{l}\text { Transversal/ } \\
\text { Descritivo }\end{array}$ & $\begin{array}{l}\text { Conhecer as características dos idosos } \\
\text { institucionalizados avaliando variáveis } \\
\text { sociodemográficas, clínicas e nível de } \\
\text { dependência funcional. }\end{array}$ & Índice de Katz \\
\hline $\begin{array}{l}\text { Indicadores } \\
\text { capacidade funcional de idosos } \\
\text { diabéticos. }\end{array}$ & Exploratório & 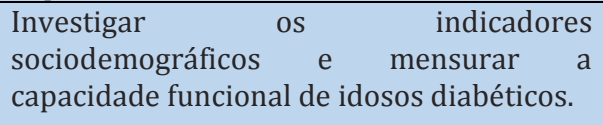 & Índice de Katz \\
\hline $\begin{array}{l}\text { Indicadores sociodemográficos e } \\
\text { capacidade funcional de idosos } \\
\text { diabéticos. }\end{array}$ & Exploratório & 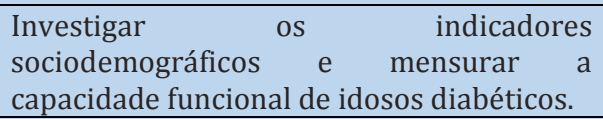 & Índice de Katz \\
\hline $\begin{array}{l}\text { O idoso institucionalizado: avaliação } \\
\text { da capacidade funcional e aptidão } \\
\text { física. }\end{array}$ & Multicêntrico & $\begin{array}{l}\text { Explorar a relação entre aptidão física e a } \\
\text { capacidade funcional de residentes em } \\
\text { Instituições de Longa Permanência para } \\
\text { Idosos de baixa renda. }\end{array}$ & Índice de Katz \\
\hline $\begin{array}{l}\text { Prevalência de incapacidade } \\
\text { funcional e dependência em idosos } \\
\text { atendidos em um Centro de Saúde- } \\
\text { Escola da Universidade de São } \\
\text { Paulo. }\end{array}$ & Transversal & $\begin{array}{l}\text { Descrever o perfil sociodemográfico de } \\
\text { idosos atendidos em um serviço } \\
\text { ambulatorial e estimar a prevalência de } \\
\text { incapacidade funcional. }\end{array}$ & Índice de Barthel \\
\hline Relação entre funcionalidade & Transversal & Analisar a relação entre funcionalidade & Índice de Barthel \\
\hline
\end{tabular}




\begin{tabular}{|c|c|c|c|}
\hline $\begin{array}{l}\text { familiar e capacidade funcional de } \\
\text { idosos dependentes no município de } \\
\text { Jequié-BA. }\end{array}$ & & $\begin{array}{l}\text { familiar quanto à adaptação, ao } \\
\text { companheirismo, ao desenvolvimento, à } \\
\text { afetividade e à capacidade funcional de } \\
\text { idosos dependentes. }\end{array}$ & \\
\hline $\begin{array}{l}\text { Validação, no Brasil, do Índice de } \\
\text { Barthel em idosos atendidos em } \\
\text { ambulatórios. }\end{array}$ & Transversal & $\begin{array}{l}\text { Validar o Índice de Barthel para idosos } \\
\text { atendidos em ambulatórios no Brasil por } \\
\text { meio da análise de confiabilidade e } \\
\text { validade. }\end{array}$ & Índice de Barthel \\
\hline \begin{tabular}{llr} 
Avaliação & da & \multicolumn{2}{c}{ capacidade } & de \\
realização & das & atividades \\
quotidianas & em idosos & residentes \\
em domicílio. & & \\
\end{tabular} & $\begin{array}{l}\text { Transversal/ } \\
\text { Descritivo }\end{array}$ & $\begin{array}{l}\text { Descrever a capacidade de realização das } \\
\text { atividades cotidianas em idosos residentes } \\
\text { em domicílio. }\end{array}$ & Índice de Barthel \\
\hline $\begin{array}{l}\text { Capacidade funcional de idosos de } \\
\text { uma comunidade rural do Rio } \\
\text { Grande do Sul. }\end{array}$ & Seccional & $\begin{array}{l}\text { Identificar a capacidade funcional de } \\
\text { idosos residentes em uma comunidade do } \\
\text { Rio Grande do Sul. }\end{array}$ & OARS \\
\hline $\begin{array}{l}\text { Morbidade e sua interferência na } \\
\text { capacidade funcional de idosos. }\end{array}$ & Seccional & $\begin{array}{l}\text { Identificar a interferência de morbidades } \\
\text { na capacidade funcional de idosos } \\
\text { atendidos em serviço ambulatorial de um } \\
\text { hospital escola de nível terciário. }\end{array}$ & OARS \\
\hline $\begin{array}{l}\text { Avaliação da capacidade funcional } \\
\text { de idosos cadastrados na Estratégia } \\
\text { Saúde da Família na comunidade do } \\
\text { Pontal da Barra, Maceió-AL. }\end{array}$ & Prospectivo & $\begin{array}{l}\text { Avaliar a capacidade de idosos cadastrados } \\
\text { na Estratégia Saúde da Família na } \\
\text { comunidade do Pontal da Barra, Maceió- } \\
\text { AL. }\end{array}$ & OARS \\
\hline $\begin{array}{llr}\begin{array}{l}\text { Fatores } \\
\text { capacidade }\end{array} & \text { determinantes } & \text { da } \\
\text { longevos. } & & \\
\end{array}$ & Transversal & $\begin{array}{l}\text { Determinar os fatores associados à } \\
\text { capacidade funcional em idosos longevos. }\end{array}$ & MIF \\
\hline $\begin{array}{l}\text { Envelhecimento ativo e sua relação } \\
\text { com a independência funcional }\end{array}$ & $\begin{array}{l}\text { Transversal/ } \\
\text { Descritivo }\end{array}$ & $\begin{array}{l}\text { Analisar os fatores determinantes para um } \\
\text { envelhecimento mais saudável e verificar o } \\
\text { grau de independência funcional de idosos } \\
\text { cadastrados em uma Unidade de Saúde da } \\
\text { Família. }\end{array}$ & MIF \\
\hline $\begin{array}{l}\text { Incapacidade funcional em mulheres } \\
\text { idosas de baixa renda. }\end{array}$ & Transversal & $\begin{array}{l}\text { Analisar a relação dos aspectos } \\
\text { sociodemográficos e referentes às } \\
\text { condições de saúde com a condição de } \\
\text { dependência na realização das atividades } \\
\text { instrumentais da vida diária. }\end{array}$ & Índice de Lawton \\
\hline
\end{tabular}

*Continuação Tabela 2.

\section{DISCUSSÃO}

Para melhor visualização, optou-se por categorizar duas áreas de discussão, descritas a seguir.

\section{- Capacidade funcional e envelhecimento}

Desde as últimas décadas do século passado, o Brasil se depara com um declínio rápido e acentuado da fecundidade, fenômeno sem precedentes na sua história e que se sobressai mesmo em comparação com outros países, seja do mundo desenvolvido, seja entre aqueles em desenvolvimento. Como aconteceu na maioria desses países, esse declínio, combinado com a queda da mortalidade, acarretou um processo de envelhecimento populacional e de aumento da longevidade da população. Apesar do processo de envelhecimento não estar, necessariamente, relacionado a doenças e incapacidades, as doenças crônicodegenerativas são frequentemente encontradas entre os idosos. Assim, a tendência atual é termos um número crescente de indivíduos idosos que, apesar de viverem mais, apresentam maiores condições crônicas. E o aumento no número de doenças crônicas está diretamente relacionado com uma maior incapacidade funcional ${ }^{6}$.

Uma das medidas efetivas nesse sentido foi a adoção da "capacidade funcional" como o novo paradigma da Política Nacional de Saúde da Pessoa por meio da Portaria n. -2.528 , de 19 de outubro de 2006. A capacidade funcional traduz-se pela capacidade do indivíduo de realizar, de maneira independente, as atividades de vida diária (AVD). A impossibilidade de fazê-lo sem auxílio constitui a dependência funcional ${ }^{7}$. Segundo a Classificação Internacional de Funcionalidade (CIF), uma doença/desordem pode gerar um déficit em um ou mais sistemas específicos do organismo, o que, por sua vez, pode levar a 
limitações no desempenho de determinadas funções. As limitações apresentadas pelos indivíduos, juntamente com sua competência em supri-las e a continência contextual que os cerca, representam os fatores determinantes do nível de incapacidade ${ }^{7,8}$.

Quando se pensa na elaboração de uma nova política de cuidado para o idoso baseada na qualidade de vida, se assume a importância de definir o conceito de capacidade funcional, isto é, a capacidade de se manter as habilidades físicas e mentais necessárias para uma vida independente e autônoma. A maioria das doenças crônicas dos idosos tem seu principal fator de risco na própria idade. No entanto, a longevidade não impede que o idoso possa conduzir sua própria vida de forma autônoma e decidir sobre seus interesses. 0 idoso que mantém sua independência e autodeterminação capacidade de o indivíduo poder exercer sua autonomia - deve ser considerado saudável, ainda que apresente uma ou mais doenças crônicas ${ }^{9,10}$.

No final da década de 1990, a Organização Mundial da Saúde (OMS) passou a utilizar o conceito de "envelhecimento ativo", buscando incluir, além dos cuidados com a saúde, outros fatores que afetam o envelhecimento. Esse conceito pode ser compreendido como o processo de otimização das oportunidades de saúde, participação e segurança, com o objetivo de melhorar a qualidade de vida à medida que as pessoas ficam mais velhas ${ }^{9}$. Ele envolve políticas públicas que promovam modos de viver mais saudáveis e seguros em todas as etapas da vida, favorecendo a prática de atividades físicas no cotidiano e no lazer, a prevenção às situações de violência familiar e urbana, o acesso a alimentos saudáveis e a redução do consumo de tabaco, entre outras medidas, que contribuirão para o alcance de um envelhecimento que signifique também um ganho substancial em qualidade de vida e saúde ${ }^{10}$.

Do ponto de vista de saúde pública, esse conceito é o mais adequado para se estruturar e viabilizar uma política de atenção à saúde do idoso. Portanto, todas as iniciativas de promoção da saúde, assistência e reabilitação em saúde devem ter como meta aprimorar, manter ou recuperar a capacidade funcional do indivíduo pelo maior tempo possível, valorizar sua autonomia e independência física e mental, excedendo o simples diagnóstico e o tratamento de doenças específicas. A dependência, física ou mental, é um fator de risco importante para a mortalidade, mais até do que as próprias doenças que levam à dependência, uma vez que nem toda pessoa doente se torna dependente. Assim, devem ser estabelecidas novas prioridades e ações de saúde que nortearão as políticas de saúde contemporâneas ${ }^{9,10}$.

É importante destacar que os extremos da funcionalidade, entendidos como diferentes níveis de desempenho e competência funcional, como aqueles presentes no envelhecimento bem-sucedido e no envelhecimento com fragilidade, estão associados a desfechos positivos e negativos, respectivamente. A funcionalidade relacionada ao envelhecimento bemsucedido está associada a maiores níveis de satisfação e bem-estar subjetivo, maior senso de autoeficácia e controle pessoal, maior rede de relações sociais, melhor saúde e independência física e mental, e a um envolvimento mais ativo com a vida, a despeito da presença de doenças crônicas. No outro extremo, a funcionalidade relacionada à fragilidade está associada a maiores níveis de mortalidade e comorbidades, pior estado de saúde, maior dependência funcional, aumento do risco de institucionalização e maior prevalência de síndromes geriátricas, como quedas, imobilismo, insuficiência cognitiva e incontinência urinária ${ }^{10,11}$.

Entre esses extremos de funcionalidade, vários níveis de desempenho podem estar presentes. Essa complexa relação entre as várias dimensões do envelhecimento torna, por vezes, difícil distinguir processos patológicos daqueles considerados como uma evolução "normal" do processo de envelhecimento. A funcionalidade representa um continuum de 
estados funcionais com vários grupos possíveis de desempenho ou, olhando sob o aspecto negativo, vários graus de comprometimento. Para os geriatras e gerontólogos, o foco na determinação das causas e no manejo do declínio funcional permanece uma questão central. Há uma associação importante e bem descrita entre o declínio funcional e a presença de doenças na determinação da fragilidade com o avançar da idade ${ }^{10-12}$.

Os trabalhos elencados evidenciam que é importante valorizar as características sociodemográficas, econômicas e de saúde dos idosos, tendo em vista as especificidades desse grupo considerando a variabilidade das condições de saúde e a qualidade de vida nas diversas faixas etárias. Destacam ainda a necessidade de os serviços e profissionais de saúde considerarem a multidimensionalidade e as peculiaridades do contexto de vida dos idosos visando à manutenção da independência, autonomia, inserção social e melhoria da qualidade de vida.

As variáveis prevalentes encontradas apontam para o gênero feminino, com idade entre 70 e 85 anos, representado por mulheres vivendo sozinhas, viúvas, de baixa renda e não alfabetizadas. Esses indicadores são apontados como relevantes para elevar a possibilidade de desenvolver incapacidade funcional.

\section{- Principais instrumentos utilizados para a mensuração da capacidade funcional em idosos}

Uma avaliação funcional pode ser constituída por vários itens, no entanto, nos mais citados, estão incluídas questões referentes à mobilidade (deambulação em distâncias determinadas, mudanças no curso da marcha, levantar-se de uma cadeira e assentar-se nela, mudanças de decúbito e transferência), às atividades básicas de vida diária (vestir-se, alimentar-se, tomar banho, entre outras), às atividades instrumentais de vida diária (utilizar meios de transporte, cozinhar, controlar finanças, usar o telefone, entre outras), além de algumas avaliações que contemplam o desempenho do indivíduo no trabalho, no ambiente social e no lazer ${ }^{13,14}$.

Entre os métodos de aplicação das avaliações funcionais, tem-se a forma do autorrelato, que mede a função física pela emissão de uma resposta do indivíduo às questões do seu funcionamento; é uma forma fácil, rápida e pouco onerosa de avaliar o paciente. Entretanto, a percepção do indivíduo sobre o seu estado geral pode, algumas vezes, influenciar suas respostas. Pequenas alterações funcionais podem, também, nesse tipo de avaliação, passar despercebidas pelos idosos. Indivíduos depressivos podem superestimar suas incapacidades, e indivíduos com alterações cognitivas, mesmo que moderadas, podem apresentar um falso desempenho nos testes. Como esses testes são questionários que necessitam da leitura dos itens, interferências em seus resultados são notadas em pacientes com baixo nível de escolaridade, deficiências na leitura e diminuição da acuidade visual ${ }^{15-17}$.

A seguir, se apresentam alguns instrumentos utilizados para avaliar a capacidade funcional de idosos. Vale ressaltar que existem muitos outros testes e baterias de testes funcionais. Sendo assim, optou-se por discutir somente os instrumentos que foram utilizados nas pesquisas dos artigos selecionados.

\section{Índex de Atividades Básicas da Vida de Diária de Katz}

O Index de Independência nas Atividades de Vida Diária desenvolvido por Sidney Katz é, ainda hoje, um dos instrumentos mais utilizados nos estudos gerontológicos nacionais e internacionais, embora tenha sido publicado pela primeira vez em 1963. $\mathrm{Na}$ década de 1950, era consenso entre os pesquisadores que o processo de envelhecimento relacionava-se com mudanças ocorridas no transcorrer do tempo que resultavam em perda progressiva das habilidades e no crescente aumento da razão de morte ${ }^{18}$.

Com base na análise dos dados obtidos, a referida equipe desenvolveu um instrumento que buscava avaliar a independência funcional dos pacientes para 
banhar-se, vestir-se, ir ao banheiro, transferir-se da cama para a cadeira e viceversa, ser continente e alimentar-se, atividades essas consideradas básicas e biopsicossocialmente integradas ${ }^{19}$.

Para o desenvolvimento de tal instrumento, era registrado o tipo de assistência recebida pelo idoso no desempenho de tais atividades. Assim, a caracterização funcional dos pacientes era obtida por meio do desempenho funcional destes e não da sua capacidade para realizar a função, ou seja, um idoso que se recusasse a desenvolver alguma das atividades citadas era considerado incapaz para a função, mesmo que fosse potencialmente capaz de realizá-la ${ }^{19,20}$.

0 Index de Independência nas Atividades de Vida Diária (AVDs) - Index de ADL desenvolvido por Sidney Katz é um instrumento de avaliação funcional muito utilizado na literatura gerontológica, tanto em nível nacional quanto internacional. Essa revisão histórica buscou mostrar o instrumento como foi concebido originalmente, seus pressupostos e embasamento teórico ${ }^{21}$.

Algumas modificações sugeridas e/ou autorizadas pelo autor também puderam ser encontradas, juntamente com as justificativas e limitações de suas aplicações, tendo, dessa forma, referências próprias que diferem da relacionada ao instrumento original. Cuidados devem ser tomados no sentido de se buscar a utilização de determinados instrumentos em sua versão original ou, em caso de modificações ou adaptações, que estas estejam validadas. Essa recomendação se deve ao fato de que algumas alterações podem impedir a comparação entre alguns estudos ou ocasionar uma análise equivocada de resultados comparativos ${ }^{22,23}$.

\section{Índice de Barthel}

Desenvolvida em 1965 para avaliar o potencial funcional e os resultados do tratamento de reabilitação dos pacientes que sofreram acidente vascular cerebral, esse teste mede o grau de assistência exigido em 10 atividades (alimentação, banho, higiene pessoal, vestir-se, controle de esfíncter, transferência cadeira e cama, deambulação e subir escadas).

Um estudo ${ }^{22}$ mostrou a validade e confiabilidade em pacientes idosos sem alterações cognitivas e com menos de 65 anos. São atribuídos pesos específicos para cada atividade proposta de acordo com a observação clínica. Na versão original, cada item é pontuado de acordo com o desempenho do paciente em realizar tarefas de forma independente, com alguma ajuda ou de forma dependente. Uma pontuação geral é formada atribuindo-se pontos em cada categoria, a depender do tempo e da assistência necessária a cada paciente. A pontuação varia de 0 a 100, em intervalos de cinco pontos, e as pontuações mais elevadas indicam maior independência. Pontuações abaixo de 50 indicam dependência em atividades de vida diária. 0 índice de Barthel tem sido aplicado em pacientes internados em unidades de reabilitação, bem como em idosos que vivem na comunidade, apresentando boa correlação com outras medidas funcionais ${ }^{24,25}$.

\section{Índice de Autocuidado de Kenny}

Trata-se de um instrumento composto de 17 tarefas distribuídas em 85 itens agrupadas em seis categorias: locomoção, transferências, atividades básicas, vestuário, higiene pessoal e alimentação. Cada item avaliado possui uma pontuação correspondente, sendo assim, atribui-se a nota zero (0) para dependência total, 1 para assistência intensiva, 2 para assistência moderada, 3 para assistência mínima e 4 quando não há necessidade de assistência.

0 estudo original envolvendo esse instrumento foi realizado em 1965 pela equipe do Instituto de Reabilitação de Kenny nos Estados Unidos e envolveu a participação de profissionais da Terapia Ocupacional, Enfermagem e Fisioterapia. 0 avanço da sofisticação da medicina trouxe a necessidade de se definir melhor a capacidade funcional e de se criar um método lógico, ordenado e sistemático para avaliar o grau de extensão das deficiências da capacidade. 0 desenvolvimento desse método facilitaria a descrição do progresso e 
compartilharia a eficácia das várias formas de tratamento 26,27 .

\section{Escala de Avaliação Funcional de Lawton e Brody}

A Escala de Lawton é um instrumento que analisa a capacidade funcional da pessoa idosa, enquanto indicador de saúde e bemestar, sendo possível determinar se o indivíduo pode viver sozinho. A capacidade funcional é definida pela presença ou não de dificuldades no desempenho de certos gestos e atividades da vida cotidiana, ou mesmo pela impossibilidade de desempenhá-los. Essa escala é dimensionada em termos da habilidade e independência para realizar determinadas atividades ${ }^{27}$. É influenciada por fatores demográficos, socioeconômicos, culturais e psicossociais que vão caracterizar o comportamento e o estilo de vida. A escala de AIVD de Lawton possui três tipos de respostas: a primeira significa independência, a segunda capacidade com ajuda, e a terceira dependência. São avaliadas as seguintes atividades: uso do telefone, locomoção usando meios de transporte, fazer compras, preparar refeições, arrumar a casa, realizar trabalhos manuais, lavar roupas, tomar medicamentos nas doses e horários corretos, cuidar das finanças. 0 escore total é de 27 pontos, sendo que a pontuação tem um significado apenas para o idoso individualmente, servindo como base para avaliação da qualidade de vida ${ }^{27-29}$.

Os instrumentos de avaliação funcional utilizados no Brasil têm sua origem em outros países, evidenciando a falta de produções nacionais. 0 processo de adaptação transcultural tem proporcionado a utilização de instrumentos criados em outras línguas e adaptados à nossa cultura, porém a avaliação das propriedades psicométricas e a adaptação das notas de corte são dados tão importantes quanto a adaptação. A confiabilidade e a validade são atributos fundamentais para a escolha de um instrumento, uma vez que comprovam a capacidade do instrumento de medir o que se propõe e a confiabilidade das informações advindas deste ${ }^{30}$.

\section{CONCLUSÃO}

A produção científica brasileira referente à capacidade funcional e os instrumentos utilizados em sua mensuração em idosos têm sido foco recente de atenção da equipe multidisciplinar de saúde e, principalmente, dos profissionais enfermeiros e fisioterapeutas.

As publicações demonstraram que, por meio da utilização de escalas e questionários, é possível identificar os fatores que limitam a capacidade funcional dos idosos. Nessa perspectiva, torna-se fundamental a atuação da enfermagem e dos demais profissionais envolvidos no cuidado ao idoso na detecção desses fatores, visto que direcionam o planejamento das ações de cuidados tanto no âmbito de prevenção quanto no de reabilitação, indo ao encontro das perspectivas da Política Nacional da Pessoa Idosa, que tem como meta manter e promover a autonomia e a independência dos indivíduos idosos.

Assim, os dados encontrados neste estudo evidenciam que a temática em questão possui um alto potencial de investigação a ser explorado. A avaliação da capacidade funcional do idoso possibilita intervir, por meio da promoção da saúde, com ações específicas que contribuam para evitar as incapacidades. Também permite ao enfermeiro e outros profissionais de saúde traçar o plano de cuidado especifico para cada idoso, com base nos resultados dos instrumentos utilizados para mensurar a capacidade funcional.

A diversificação do uso de escalas de avaliação da capacidade funcional do idoso, quando trabalhada criticamente, possui aspecto positivo. 0 campo de conhecimento e da prática da avaliação funcional se constrói de forma cumulativa. Assim, a escolha de uma ou mais escalas de avaliação funcional deve ser criteriosa, levando também em consideração a população-alvo a ser avaliada.

\section{REFERÊNCIAS}

1. Instituto Brasileiro de Geografia e Estatística (IBGE). Diretoria de Pesquisas Coordenação de População e 
Indicadores Sociais. Síntese dos indicadores sociais: uma analise das condições de vida da população brasileira em 2010. Rio de Janeiro: IBGE; 2010. Idosos; p.191-197.

2. Ministério da Saúde (Br). Envelhecimento e saúde da pessoa idosa. Brasília: Ministério da Saúde; 2006. 192 p. Série A. Normas e Manuais Técnicos. Cadernos de Atenção Básica; n 19.

3. Shubert TE, Schrodt LA, Mercer VS, Busby-Whitehead J, Giuliani CA. Are scores on balance screening tests associated with mobility in older adults? J Geriatr Phys Ther. 2006; 29 (1):33-9.

4. Paschoal SMP. Autonomia e independência. In: Papaléo-Netto M, organizador. Gerontologia: a velhice o envelhecimento em visão globalizada. São Paulo: Atheneu; 2008. p. 311-323.

5. Gil AC. Como elaborar projetos de pesquisa. 5 ed. São Paulo: Atlas; 2010. p. 25-44.

6. Torres GV, Reis LA, Fernandes MH, Alves GS. Avaliação da capacidade de realização das atividades quotidianas em idosos residentes em domicílio. Rev Baiana Saúde Pública. 2009; 33(3):466-75.

7. Bispo EPF, Rocha MCG, Rocha MFMR. Avaliação da capacidade funcional de idosos cadastrados na Estratégia Saúde da Família na comunidade do Pontal da Barra, Maceió-AL. Cad Ter Ocup UFSCar. 2012; 20(1):817.

8. Nunes DP, Nakatani AYK, Silveira EA, Bachion MM, Souza MR. Capacidade funcional, condições socioeconômicas e de saúde de idosos atendidos por Equipes de Saúde da Família de Goiânia (GO, Brasil). Ciênc Saúde Coletiva. 2010; 15(6):2887-98.

9. Veras R. Envelhecimento populacional contemporâneo: demandas, desafios e inovações. Rev Saúde Pública. 2009; 43(3):548-54.

10. Ferreira OGL, Maciel SC, Costa SMG, Silva AO, Moreira MASP. Envelhecimento ativo e sua relação com a dependência funcional. Texto e Contexto Enferm. 2012; 21(3):513-8.

11. Nogueira SL, Ribeiro RCL, Rosado LEFPL, Franceschini SCC, Ribeiro AQ, Pereira ET. Fatores determinantes da capacidade funcional em idosos longevos. Braz. J. Phys. Ther. 2010; 14(4):322-9.

12. Xavier GS, Brito GEG, Oliveira EA, Carvalho DB, Rolim IB, Lucena EMF. Capacidade funcional de idosos adscritos à Estratégia Saúde da Família no município de João Pessoa-PB. Rev Bras Ciênc Saúde. 2011; 15(3):28794.

13. Santos AAS, Pavarini SCI. Funcionalidade de idosos com alterações cognitivas em diferentes contextos de vulnerabilidade social. Acta Paul Enferm. 2011; 24(4):520-6.

14. Rigo II, Paskulin LMG, Morais EP. Capacidade funcional de idosos de uma comunidade rural do Rio Grande do Sul. Rev Gaúch Enferm. 2010; 31(2):254-61.

15. Virtuoso JSJ, Guerra RO. Incapacidade funcional em mulheres idosas de baixa renda. Ciênc Saúde Coletiva. 2011; 16(5):2541-8.

16. Aires M, Paskulin LMG, Morais EP. Capacidade funcional de idosos mais velhos: estudo comparativo em três regiões do Rio Grande do Sul. Rev. Latinoam. Enfer. 2010; 18(1):11-7.

17. Katz S, Chinn AB. Multidisciplinary studies of illness in aged persons II: a new classification of functional status in activities of daily living. J Chronic Dis. 1959; 9(1):55-62.

18. Katz S, Downs TD, Cash HR, Grotz RC. Progress in development of the index of ADL. Gerontologist.1970; 10(1):20-30.

19. Smanioto FN, Haddad MCFL. Índice de Katz aplicado a idosos institucionalizados. Rev RENE. 2011; 12(1):1823.

20. Brito TRP, Pavarini SCI. Relação entre apoio social e capacidade funcional de idosos com alterações cognitivas. Rev Latinoam. Enfer. 2012; 20(4):677-84.

21. Minosso JSM, Amendola F, Alvarenga MRM, Oliveira MAC. Validação, no Brasil, do Índice de Barthel em idosos atendidos em ambulatórios. Acta Paul Enferm. 2010; 23(2):218-23.

22. Minosso JSM, Amendola F, Alvarenga MRM, Oliveira MAC. Prevalência de incapacidade funcional e dependência em idosos atendidos em um Centro de Saúde-Escola da Universidade de São Paulo. Cogitare Enfer. 2010; 15(1):12-8.

23. Lisboa CR, Chianca TCM. Perfil epidemiológico, clínico e de independência funcional de uma população idosa institucionalizada. Rev Bras Enferm. 2012; 65(3):482-7.

24. Lawton MP, Brody EM. Assessment of older people: self-maintaining and instrumental activities of daily living. Gerontologist. 1969; 9(3):179-86.

25. Rodrigues RAP, Scudeller PG, Pedrazzi EC, Schiavetto FV, Lange C. Morbidade e sua interferência na capacidade funcional de idosos. Acta Paul Enferm. 2008; 21(4):643-8.

26. Duca GFD, Thumé E, Hallal PC. Prevalência e fatores associados ao cuidado domiciliar a idosos. Rev Saúde Publica. 2011; 45(1):113-20.

27. Gonçalves LHT, Silva AH, Mazo GZ, Benedetti TRB, Santos SMA, Marques S, Rodrigues RAP et al. O idoso institucionalizado: avaliação da capacidade funcional e aptidão física. Cad Saúde Pública. 2010; 26(9):1738-46.

28. Fernandes MGM, Pereira MA, Honorato MM, Fernandes BM. Indicadores sociodemográficos e capacidade funcional de idosos diabéticos. Rev Bras Ciênc Saúde. 2011; 15(1):57-64.

29. Perracicini MR, Fló CM, Guerra RO. Funcionalidade e envelhecimento. In: Perracini MR, Fló CM, organizadores. Funcionalidade e envelhecimento. Rio de Janeiro: Guanabara Koogan; 2011. p.24-43.

30. Canon MBF, Novelli MMPC. Estudo dos instrumentos de avaliação funcional em demência utilizados no Brasil. Rev Ter Ocup. 2012; 23(3):253-62.

CONTRIBUIÇõES
Gerson de Souza e Santos Isabel Cristina
Kowal Olm Cunha tiveram iguais
contribuições na concepção e delineamento
do estudo, análise e interpretação dos dados
e, redação do manuscrito.

